\title{
Targeting the B-cell lymphoma 2 anti-apoptotic proteins for cervical cancer treatment
}

\author{
Siti Fairus Abdul Rahman, Benedict Shi Xiang Lian² \& Nethia Mohana-Kumaran*,1 (iD) \\ ${ }^{1}$ School of Biological Sciences, Universiti Sains Malaysia, 11800 Penang, Malaysia \\ ${ }^{2}$ Division of Biological Science, Graduate School of Science \& Technology, Nara Institute of Science \& Technology (NAIST), Ikoma, \\ Nara 630-0101, Japan \\ *Author for correspondence: nethiakumaran@usm.my
}

The B-cell lymphoma 2 (BCL-2) anti-apoptotic proteins have become attractive therapeutic targets especially with the development of BH3-mimetics which selectively target these proteins. However, it is important to note that expression levels of the anti-apoptotic proteins and their relevance in inhibiting apoptosis varies between different cell lineages. This addiction to certain anti-apoptotic proteins for survival, can be determined with various techniques and targeted effectively with selective BH3-mimetics. Studies have highlighted that anti-apoptotic proteins BCL-XL and MCL-1 are crucial for cervical cancer cell survival. Co-targeting BCL-XL and MCL-1 with selective BH3-mimetics yielded promising results in cervical cancer cell lines. In this review, we focus on the expression levels of the anti-apoptotic proteins in cervical cancer tissues and how to possibly target them with BH3-mimetics.

First draft submitted: 22 April 2020; Accepted for publication: 23 June 2020; Published online: 26 July 2020

Keywords: $\mathrm{BCL}-2$ selective inhibitors $\bullet \mathrm{BCL}-\mathrm{XL}$ selective inhibitors $\bullet \mathrm{BH}$-mimetics $\bullet$ cervical cancer $\bullet \mathrm{MCL}-1$ selective inhibitors

Persistent infection with high-risk human papillomavirus (hrHPVs) are the causative agent for the development of cervical cancer. HPV transmits primarily through sexual activities. There are 200 identified HPV types and 12 have been identified as carcinogenic with hrHPV-16 and hrHPV-18 account for 50 and 10\% cervical cancer cases, respectively [1]. These viruses can be detected in $99.7 \%$ of patients diagnosed with cervical cancer worldwide [2]. Other HPV subtypes namely HPV-31, -33 and -45 have been identified in cervical cancer but less frequently compared with HPV-16 and HPV-18 [1,3]. The estimated number of new cases of cervical cancer in 2018 was 568,847 and the number of deaths were 311,365 [4]. The incidences and mortality in less developed countries are higher compared with high-income countries due to reduced access to screening and the high cost of the HPV vaccine [5].

Current cervical cancer treatment consists of surgery, radiotherapy or chemotherapy [6,7]; treatment resistance, particularly in advance and recurrent cases of cervical cancer, remains a challenge. Deeper understanding of signaling pathways and gene aberrations in cervical cancer pathogenesis, have assisted in identifying potential molecular targets for therapy and have led to the use of targeted therapies for the treatment of cervical cancer either as single agents or combination with chemotherapeutic drugs. Thus far, bevacuzimab (Avastin ${ }^{\circledR}, \mathrm{Mvasi}^{\mathrm{TM}}-$ targets VEGF) and immune checkpoint inhibitor pembrolizumab (Keytruda ${ }^{\circledR}$ - targets the PD-L1 protein) are the only targeted therapies approved by the US FDA for the treatment of advance and recurrent cervical cancer [7]. Bevacuzimab is used in combination with chemotherapeutic drugs namely cisplatin, paclitaxel and carboplatin to treat patients with advance and recurrent cervical cancer. Pembrolizumab received accelerated approval from FDA in July 2018 for the treatment of patients diagnosed with advance recurrent cervical cancer [7]. Approval of targeted therapies for the management of cervical cancer has opened new treatment avenues for patients who otherwise had limited treatment options once they developed resistance to standard treatment.

Future Medicine 
The BCL-2 family of proteins, the regulators of the intrinsic apoptosis pathway, emerged as attractive targets for cancer treatment. Attention to these proteins increased with the emergence of small molecule inhibitors known as BCL-2 homology 3 (BH3)-mimetics which potently and selectively inhibit certain members of the BCL-2 family of proteins and induce apoptosis in cancer cells [8]. Targeting the BCL-2 family of proteins with BH3-mimetics for cervical cancer treatment has not been explored extensively despite studies reporting high expression levels of certain members of the family in cervical cancer tissues. Targeting these proteins could be a new treatment approach for cervical cancer management. Here we give an overview of the intrinsic apoptosis pathway, discuss the expression levels of the BCL-2 family of proteins in cervical cancer and approaches to target these proteins with BH3-mimetics for cervical cancer management.

\section{Apoptosis pathways}

Apoptosis is a form of programmed cell death for neat disposal of aged, damaged or diseased cells. Apoptosis plays a major role in normal developmental processes, including eliminating cells during tissue morphogenesis and in the elimination of inactive and self-activating cells during lymphopoiesis. Abrogation of apoptosis pathways can lead to manifestation of cancer or degenerative disease. Cells activate apoptosis following diverse stress signals, namely activation of oncogenes, ultraviolet radiation, growth factor deprivation and DNA damage caused by drugs. Apoptosis can be initiated through the intrinsic (also known as the mitochondrial) pathway or the extrinsic (also known as death receptor) pathway [9].

The intrinsic pathway can be activated following diverse stress signals such as hypoxia, nutrient deprivation, DNA damage caused by chemotherapeutic agents and targeted therapies (Figure 1). Once the stress is sensed, the pro-apoptotic BCL-2 proteins neutralize the anti-apoptotic BCL-2 proteins. Inactivation of the anti-apoptotic BCL-2 proteins leads to translocation of BAX from the cytoplasm to the outer mitochondrial membrane (OMM). At the OMM, BAX oligomerizes with BAK to induce mitochondrial outer membrane permeabilization (MOMP) leading to release of mitochondrial cytochrome c. Cytochrome c interacts with the monomeric cytosolic protein, APAF1, initiating the formation of apoptosome, which activates initiator caspase-9. Activated caspase-9 subsequently activates caspases-3 and -7 , which in turn activate apoptosis (Figure 1) [8].

The extrinsic pathway is activated when ligands namely FASL, TNF and TRAIL activate their respective death receptors (DR). The DRs are situated at the plasma membrane and the members include the TRAIL receptor 1 (TRAILR1, also known as DR4 and TNFRSF10A), TRAIL2 (also known as DR5 and TNFRSF10B), FAS (also known as CD95 and APO1) and TNFR1 (also known as TNFRSF1A). These DRs contain a cytosolic 'death domain' and through this death domain, they interact with the other cytosolic proteins. Once a ligand binds to its respective DR, the DR recruits the cytosolic FADD (FAS-associated death domain protein) and caspases- 8 and -10. Activation of caspase- 8 leads to activation of the executioner caspases such as caspases-3 and -7 (Figure 1). The executioner caspases then initiate the proteolytic mechanics of apoptosis in cells [10]. The extrinsic pathway crosstalks with the intrinsic pathway through the actions of caspase-8. Caspase-8 truncates and activates $\mathrm{BH} 3$-only protein BID of the intrinsic apoptosis pathway to truncated form BID [11]. Thereafter, the pathway leading to activation of apoptosis follows the intrinsic pathway (Figure 1).

\section{The BCL-2 family proteins}

The BCL-2 family members strictly regulate the induction of MOMP. The protein family is divided based on their structures and BH domains. The anti-apoptotic proteins BCL-2, BCL-XL, BCL-W, MCL-L and BFL-1/A1 contain four $\mathrm{BH}$ domains, $\mathrm{BH} 1-\mathrm{BH} 4$ [12]. The pro-apoptotic effector proteins, BAX, BAK and BOK contain 3-4 BH domains [13-15]. The pro-apoptotic BH3-only proteins, BIM, BID, PUMA, BAD, NOXA, HRK, BMF and BIK only possess the $\mathrm{BH} 3$ domain [16-22]. The $\mathrm{BH} 3$-only proteins utilize the $\mathrm{BH} 3$ domain to facilitate interaction with members of the other subgroups. Excluding BH3-only proteins BFL-1, BAD, BID, PUMA and BMF, all other proteins contain a transmembrane domain to facilitate association with the OMM [23].

Unlike the other BCL-2 family members, where expressions are subject to tissue specific and stimulus induced modulation, BAX and BAK are ubiquitously expressed. BAX normally resides in the cytoplasm and translocate to the OMM when it is activated by the BH3-only proteins [24]. Once activated BAX inserts into the OMM and oligomerizes with BAK to induce MOMP. BAX and BAK can be directly activated by $\mathrm{BH} 3$-only proteins (Figure 2) known as activators, namely BIM [18,25] and BID [20] and to a lesser extent by PUMA and NOXA [26-28]. The $\mathrm{BH} 3$ domain of the activator $\mathrm{BH} 3$-only proteins induce an allosteric change in the effector proteins which results in the formation of high-ordered oligomers that form pores in the in the mitochondrial outer membrane to 


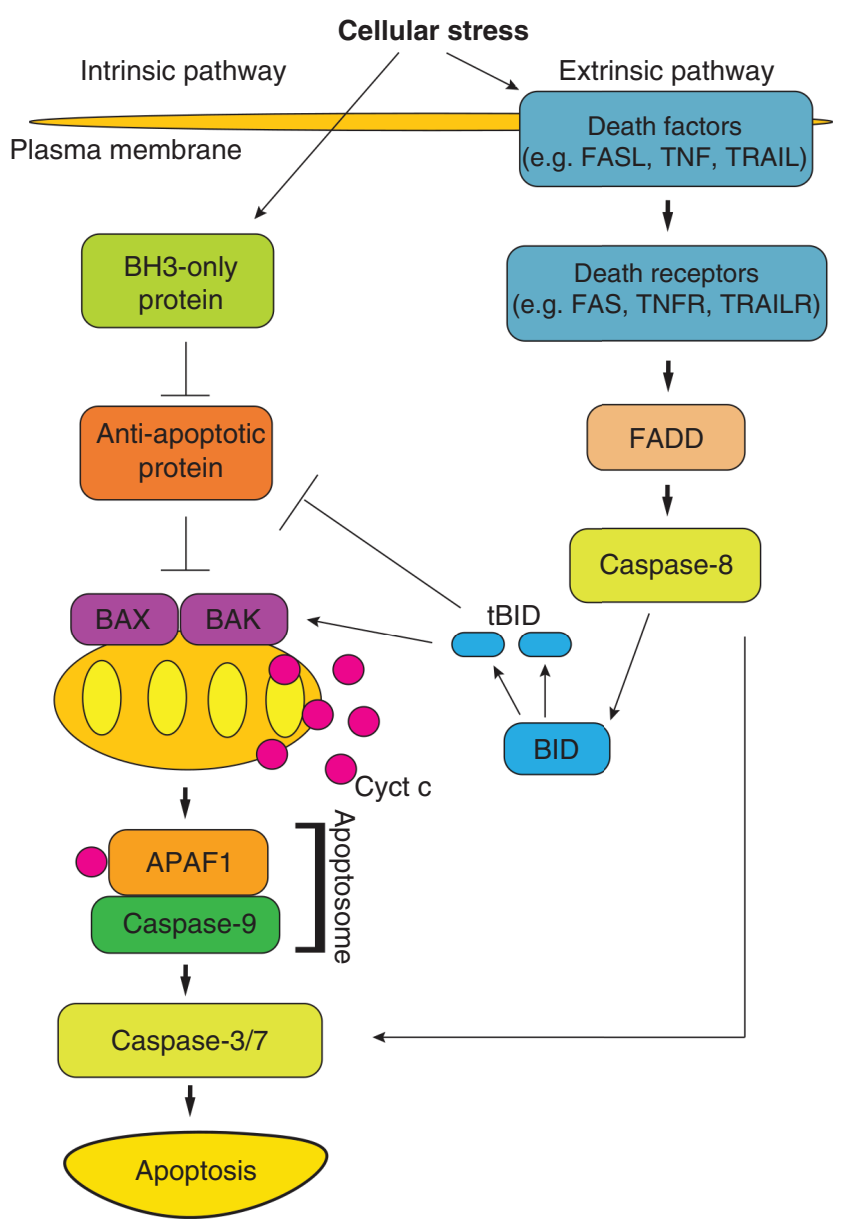

Figure 1. The apoptosis pathways. Apoptosis is activated by various cellular stress signals. For example, in response to therapy, the BH3-only pro-apoptotic proteins inhibit the anti-apoptotic proteins. This leads to oligomerization of $B A X$ and BAK which result in mitochondrial outer membrane permeabilization. This results in release of cytochrome-c. Cytochrome-c forms a complex with APAF1 and procaspase-9, which leads to the activation of caspase-9. Caspase- 9 activates executioner caspase- 3 and -7 , resulting in apoptosis. The extrinsic pathway is activated when ligands activate their respective death receptors which leads to activation of caspase- 8 . Caspase- 8 activates executioner caspase- 3 and -7 , resulting in apoptosis. Crosstalk between the intrinsic and the extrinsic pathway is mediated through the BH3-only protein BID. Activation of caspase-8 leads to cleavage of BID to truncated form BID. Truncated form BID activates the intrinsic pathway by either inhibiting the anti-apoptotic proteins or directly activating the effector pro-apoptotic proteins BAX and BAK.

BH3: B-cell lymphoma 2 homology 3.

cause MOMP [29-31]. The anti-apoptotic proteins prevent MOMP by either directly binding to the $\mathrm{BH} 3$ domain of activator BH3-only proteins (Figure 2), like BIM, BID and PUMA [32-34] or directly binding to the activated monomeric forms of the effector pro-apoptotic proteins, BAX and BAK (Figure 2), thus preventing BAX and BAK oligomerization [35-37].

The remaining members of the $\mathrm{BH} 3$-only proteins are known as the sensitizers. These proteins can lead to MOMP by two mechanisms. The sensitizer $\mathrm{BH} 3$ domain binds to anti-apoptotic proteins, replacing activators, which activate the effectors and induce MOMP (Figure 2) [32]. Another mechanism would be sensitizers replacing the monomeric effector protein BAX or BAK that are inhibited by an anti-apoptotic protein (Figure 2). The sensitizer $\mathrm{BH} 3$-only proteins exhibit distinct binding profiles toward the anti-apoptotic proteins. NOXA specifically inhibits MCL-1 and BFL-1; BAD and BMF inhibit BCL-2, BCL-XL and BCL-w; BIK and Hrk inhibit BCL-XL, BCL-w and BFL-1 (Figure 3) [28,32,34,38]. BH3-only proteins BIM, BID and PUMA, on the other hand, are promiscuous 
Growth factor deprivation, oncogene activation, chemotherapeutic drugs, targeted therapies, ultraviolet radiation

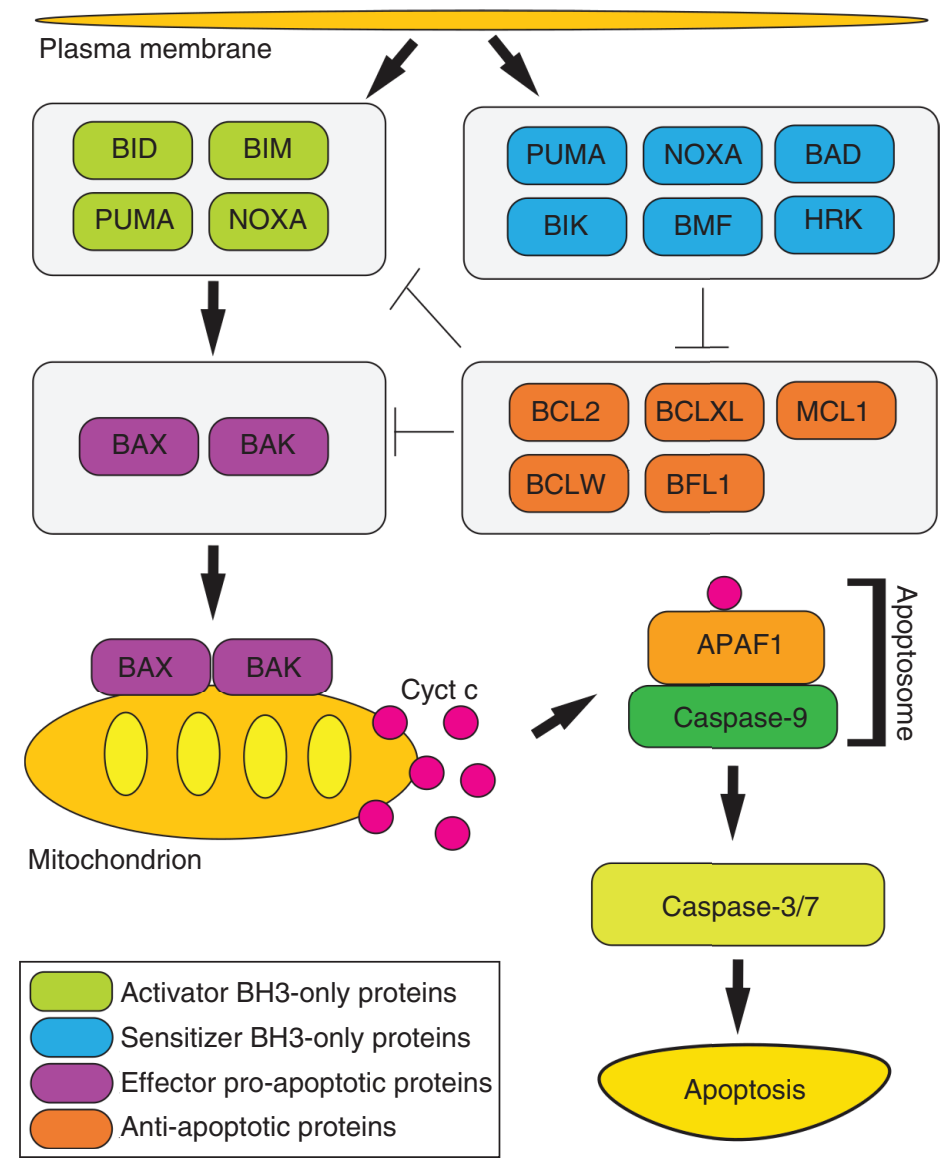

Figure 2. The BCL2 family proteins. In response to drug treatment, for example, the BH3-only activators directly activate the pro-apoptotic effector proteins. Compared with BIM and BID, PUMA and NOXA are weaker activators. The anti-apoptotic proteins either sequester the $\mathrm{BH} 3$-only activators or effector proteins to prevent apoptosis. The $\mathrm{BH} 3$-only sensitizers selectively inhibit the anti-apoptotic proteins to activate apoptosis.

BH3: B-cell lymphoma 2 homology 3.

inhibitors of the anti-apoptotic proteins (Figure 3) [39]. The selective binding profile of the BH3-only proteins toward the anti-apoptotic proteins forms the basis for designing BH3 mimetics [40].

Abbvie (previously Abbott Laboratories, IL, USA) developed the first BH3-mimetic ABT-737 that targets antiapoptotic proteins BCL-2, BCL-XL and BCL-w [41]. The second-generation BH3-mimetic ABT-263 (Navitoclax) shares the same binding profile as ABT-737 (Figure 3) but with improved pharmacological properties [42]. Unlike ABT-737, ABT-263 entered clinical trials [43,44]. However, ABT-263 causes dose-dependent thrombocytopenia as result of BCL-XL inhibition. This resulted in the emergence of BCL-2 selective inhibitor, ABT-199 (Venetoclax; Figure 3) by Abbvie, Genentech (CA, USA) and The Walter Eliza Hall Institute (VIC, Australia) [45]. Venetoclax (VENCLEXTA, Abbvie Inc. and Genentech Inc.) was approved by FDA for chronic lymphocytic leukemia in 2016. There were number of highly potent and selective BCL-XL inhibitors namely WEHI-539, A-1155463 and A-1331852 (Figure 3) [46-48] were also developed but these compounds have not entered clinical trials. Servier (Suresnes, France) published their first MCL-1 inhibitor S63845 in 2016 but this compound did not enter clinical trials [49]. Recently, a number of selective MCL-1 inhibitors have entered clinical trials namely S64315/MIK665, codeveloped by Servier and Novartis (Basel, Switzerland) [50], AMG-176 [51] and AMG-397 by Amgen (CA, USA) [50] and more recently AZD5991 by AstraZeneca (Cambridge, UK; Figure 3) [52,53]. So far, there are no $\mathrm{BH} 3$ mimetics reported for anti-apoptotic protein BFL-1. 


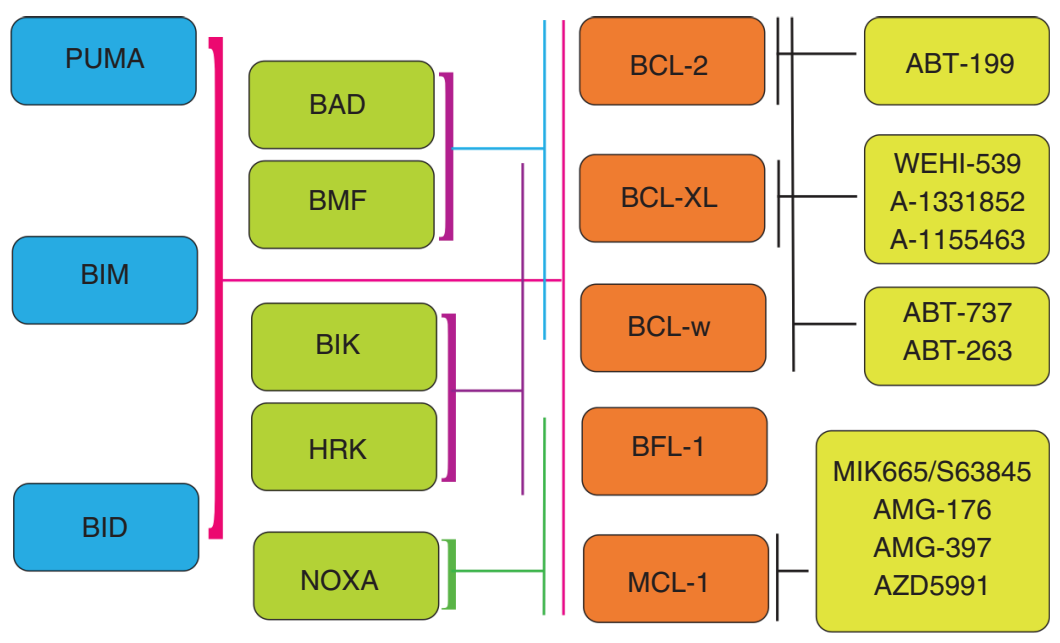

Figure 3. The left side of the image shows that BH3-only pro-apoptotic proteins have distinct specificities for the anti-apoptotic proteins. BH3-only proteins PUMA, BID and BIM are promiscuous inhibitors of the anti-apoptotic proteins unlike the other $\mathrm{BH} 3$-only proteins. The right-side of the image shows $\mathrm{BH}$-mimetics and their respective targets.

BH3: B-cell lymphoma 2 homology 3.

\section{Adaptation of cancer cells to certain BCL-2 family proteins for survival}

One way that cancer cells evade apoptosis, triggered by oncogenesis or drug treatment is via overexpressing anti-apoptotic BCL-2 proteins [54]. Hence, even if treatment is effective to activate pro-death signaling either by upregulating pro-apoptotic protein BIM or effector proteins BAX or BAK, cancer cells can survive the cytotoxic insult by sequestering these proteins with anti-apoptotic proteins [12]. However, this adaptation to anti-apoptotic proteins can vary from tumor to tumor, hence it is necessary to determine which anti-apoptotic(s) the tumor of interest depend for survival. For example, different cases of multiple myeloma rely on either BCL-2 or BCL-XL or MCL-1 or combination of these proteins for survival. Hence, they have to be treated differently from each other $[55,56]$. Acute myeloid leukemia cells developed resistance to inhibition of BCL-2, by upregulating BCL-XL and MCL-1. Co-inhibition of BCL-XL and MCL-1 resensitized acute myeloid leukemia to ABT-199, which inhibited BCL-2 [57]. Co-inhibition of BCL-2 and BCL-XL/MCL-1 killed chronic lymphocytic leukemia cells, indicating that the cells rely on multiple anti-apoptotic proteins for survival [58]. Moreover, dual inhibition of MCL-1 and BCL-2 killed Acute T lymphoblastic leukemia (T-ALL) cells in vitro and in vivo [59].

\section{Contribution of the BCL-2 anti-apoptotic proteins for cervical cancer progression}

Initial attention focused on anti-apoptotic protein BCL-2, since BCL-2 was the best-characterized member of the BCL-2 family of proteins. The expression of BCL-2 for cervical cancer progression was mainly evaluated by immunohistochemistry (IHC). There were number of studies which investigated the expression of BCL-2 in different grades of CIN (cervical intraepithelial neoplasia - there are three CIN grades namely CIN 1, CIN 2 and CIN 3) lesions and invasive squamous cervical carcinoma tissue sections. Using IHC, expression of BCL-2 was reported to increase with the rising grade of CIN [60,61], although only one study showed significance increase of BCL-2 in different CIN grades [60]. Contrary to these studies, BCL-2 expression was reported to decrease with increasing grades of CIN in one study. However, the difference reported was not significant [62].

The contribution of anti-apoptotic protein BCL-2 to cervical cancer progression is debatable. Using IHC, expression of BCL-2 was reported to significantly decrease with the increase of cervical cancer clinical stage [63]. In contrast, other groups have shown the opposite relationship between BCL-2 expression and cervical cancer progression. These studies reported that there was a trend that tumors at advanced stage showed higher expression of BCL-2 compared with tumors at early stage, however this was statistically insignificant [62,64]. However, one group reported that elevation in the expression level of BCL-2 significantly correlated with the increase of cervical cancer 
clinical stage. Moreover, this study reported, high expression level of BCL-2 in cervical cancer tissues compared with normal cervix, chronic cervicitis and CIN [65].

Association between the level of BCL-2 and cervical cancer tissue differentiation is controversial. A number of studies reported that well differentiated and moderately differentiated cervical cancer tissues were BCL-2-positive, but the difference in the levels of BCL-2 in these tissues were insignificant [62-64]. Similar to these studies, another study reported that the expression levels of BCL-2 in moderately differentiated tissues and poorly differentiated tissues were insignificant [65]. On the contrary, one study reported that BCL-2 was more frequently expressed in well-differentiated tumors compared with poorly differentiated tumors and the association between the level of BCL-2 and tumor differentiation was significant [66]. These contradictory findings question the importance of BCL-2 for cervical cancer survival.

There are limited evidence that anti-apoptotic proteins MCL-1 and BCL-XL are upregulated in cervical cancer tissues and these molecules could be potential targets for cervical cancer therapy. One group reported elevated expression of MCL-1 in cervical cancer tissues compared with normal epithelial tissues, as measured by IHC, later confirmed the findings with western blotting. Furthermore, high expression of MCL-1 was significantly associated with histological grade, tumor size, lymph node involvement and poorer prognosis [67].

Immunochemistry performed on 45 cervical cancer tissues and 21 normal cervical tissues revealed high expression of BCL-XL in cervical cancer tissues compared with normal tissues. Elevated level of BCL-XL was significantly associated with lymph node metastasis, tumor size, differentiation grade and clinical stage of cervical cancer [68]. The same study also investigated the expression of BCL-2 in the tissues and reported that expression of BCL2 significantly decreased with the increase of cervical cancer clinical stage. Moreover, there were no significant relationship between BCL-2 expression and lymph node metastasis, tumor size or differentiation grade of cervical cancer [68]. Based on these limited studies, there is a possibility that MCL-1 and BCL-XL could be potential biomarkers and therapeutic targets for cervical cancer management.

PubMed literature search only resulted in one research article reporting on RNA expression of $B F L-1$ in one ( $\mathrm{SiHa}$ ) out of the three cervical cancer cell lines studied [69]. Thus far there are no evidences on the expression level of anti-apoptotic protein BFL-1 in normal cervical epithelial tissues or cervical cancer tissues. Moving forward, investigations on the expression level of BFL-1 in cervical cancer tissues are important as this protein could be a potential biomarker. Moreover, gene knockout studies using the CRISPR/Cas9 system would be useful to determine the role of BFL-1 for cervical cancer cell survival. These suggested studies may provide insights on the possibility of targeting BFL-1 in cervical cancer treatment in the future.

\section{Targeting cervical cancer with BH3-mimetics}

Cancer cells develop a dependency on either one specific anti-apoptotic protein or more than one anti-apoptotic protein for survival due to several factors such as tissue of origin, oncogenes that are involved in driving malignant transformation, selective pressures exerted by drugs or composition of the tumor stroma [70]. Leukemia and lymphomas are mostly addicted to a single anti-apoptotic protein but the survival of solid tumors is often dependent on multiple anti-apoptotic proteins $[49,51,71]$. Hence, identifying anti-apoptotic proteins that a particular cancer cell population is addicted to is pivotal so that appropriate $\mathrm{BH} 3$-mimetics can be used to activate apoptosis in cells. The 'dynamic BH3 profiling' technique [12] or the inducible CRISPR/Cas9 platform [72] could be used as a strategy to identify dependencies on BCL-2 anti-apoptotic proteins in tumor cells. Given that, BH3-mimetics are highly selective on the targets that they inhibit, dependencies on BCL-2 anti-apoptotic proteins can be also determined by treating cancer cells with different $\mathrm{BH} 3$-mimetics $[49,73]$.

So far there are no publications reporting on the sensitivity of cervical cancer cell lines to BH3-mimetics except for studies published by our group. There are also no clinical trials of BH3-mimetics reported for cervical cancer (Table 1). In our first study, cervical cancer cell lines were treated with different BH3-mimetics, in order to identify which anti-apoptotic proteins that the cells depended for survival. Cervical cancer cell lines CaSki, SiHa and C33A were treated to single agent and combination of ABT-263 and MCL-1 selective inhibitor A-1210477. All three cell lines were resistant to single agent treatment of ABT-263 indicating that inhibition of BCL-2/BCL-XL are not sufficient to kill cells. The cell lines were also insensitive to single agent treatment of A-1210477 indicating that MCL-1 alone does not dictate the survival of these cell lines. Combination of ABT-263 and A-1210477 synergistically inhibited cell proliferation in all cell lines tested [74]. However, given that ABT-263 exhibits high affinity toward BCL-2 and BCL-XL, it was unclear whether the effect of ABT-263 was driven by BCL-2, BCL-XL or both proteins. In our subsequent study, BCL-2 selective inhibitor ABT-199 and BCL-XL selective inhibitor 


\section{Table 1. Compounds, their respective chemical structures, targets and phase of clinical development.}

Compound and chemical structure<smiles>CN(C)CC[C@H](CSc1ccccc1)Nc1ccc(NC(=O)c2ccc(N3CCN(Cc4ccccc4-c4ccc(Cl)cc4)CC3)cc2)cc1[N+](=O)[O-]</smiles>

ABT-737<smiles>CC1(C)CCC(c2ccc(Cl)cc2)=[C+](CN2CCN(c3ccc(C(=O)NS(=O)(=O)c4ccc(N[C@H](CCN5CCOCC5)CSc5ccccc5)c(S(=O)(=O)C(F)(F)F)c4)cc3)CC2)C1</smiles>

Navitoclax (ABT-263)

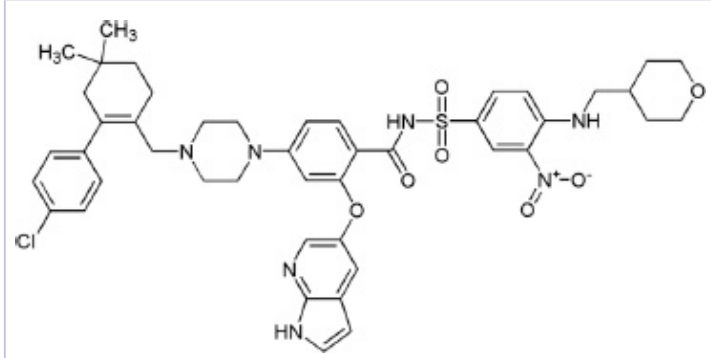

Venetoclax (ABT-199)

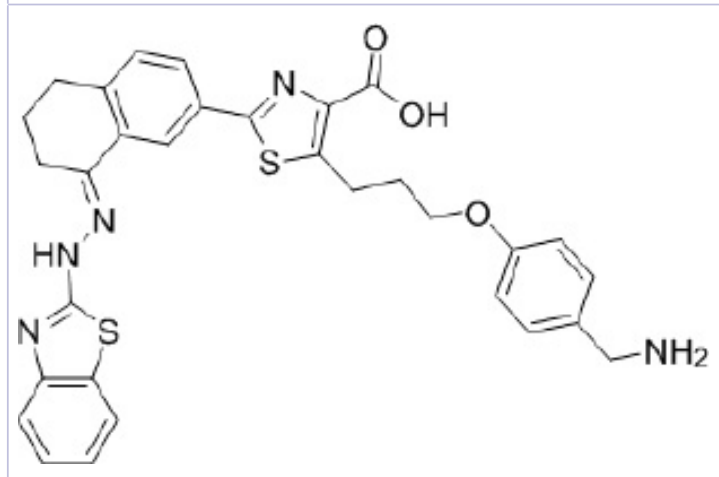

WEHI-539

Abbvie/Genentech

BCL-2

Clinical trials (source:

www.clinicaltrials.gov)

Ref.

Abbvie

BCL-2, BCL-XL and BCL-W

clinical trials for cervical cancer
Abbvie

BCL-2, BCL-XL and BCL-W

cance

Only in vitro work reported in

combination with A-1210477
No clinical trials for cervical

cancer

[74]

Only in vitro work reported in

combination with $\mathbf{5} 63845$
The Walter and Eliza Hall $\quad$ BCL-XL Institute of Medical

Research
No clinical trials for cervical cancer

BCL-2: B-cell lymphoma 2. 


\section{Table 1. Compounds, their respective chemical structures, targets and phase of clinical development (cont.).}

Compound and chemical structure

Company/institution

Abbvie

$\square$

(c)

A-1155463<smiles>Cc1c(-c2ccc(N3CCc4cccc(C(=O)Nc5nc6ccccc6s5)c4C3)nc2C(=O)O)cnn1CC12CC3CC(CC(C3)C1)C2</smiles>

A-1331852<smiles>Cc1nn(C)c(COc2ccc(N3CCN(S(=O)(=O)N(C)C)CC3)cc2)c1-c1cccc2c(CCCOc3cccc4ccccc34)c(C(=O)O)n(CCN3CCOCC3)c12</smiles>

A-1210477

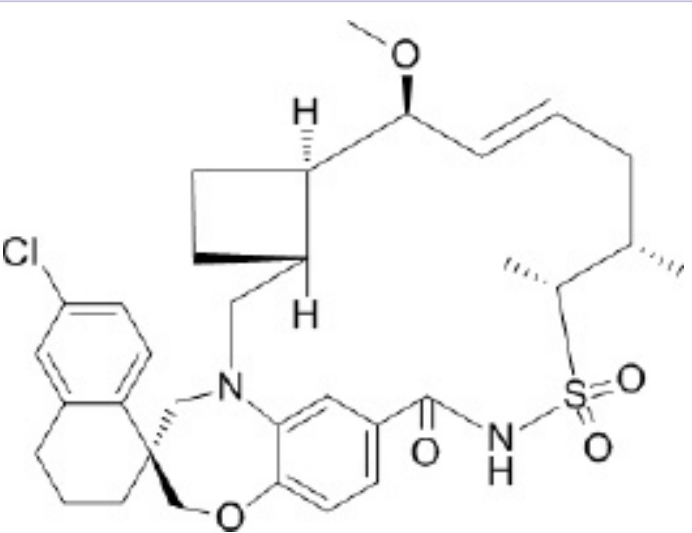

AMG-176

BCL-2: B-cell lymphoma 2.
Clinical trials (source: www.clinicaltrials.gov)

No clinical trials for cervical cance
Abbvie

Abbvie

MCL-1

o clinical trials for cervica

cancer

Only in vitro work reported in

combination with 563845
No clinical trials for cervical

cancer

No clinical trials for cervica

cancer

MCL-1 
Table 1. Compounds, their respective chemical structures, targets and phase of clinical development (cont.)

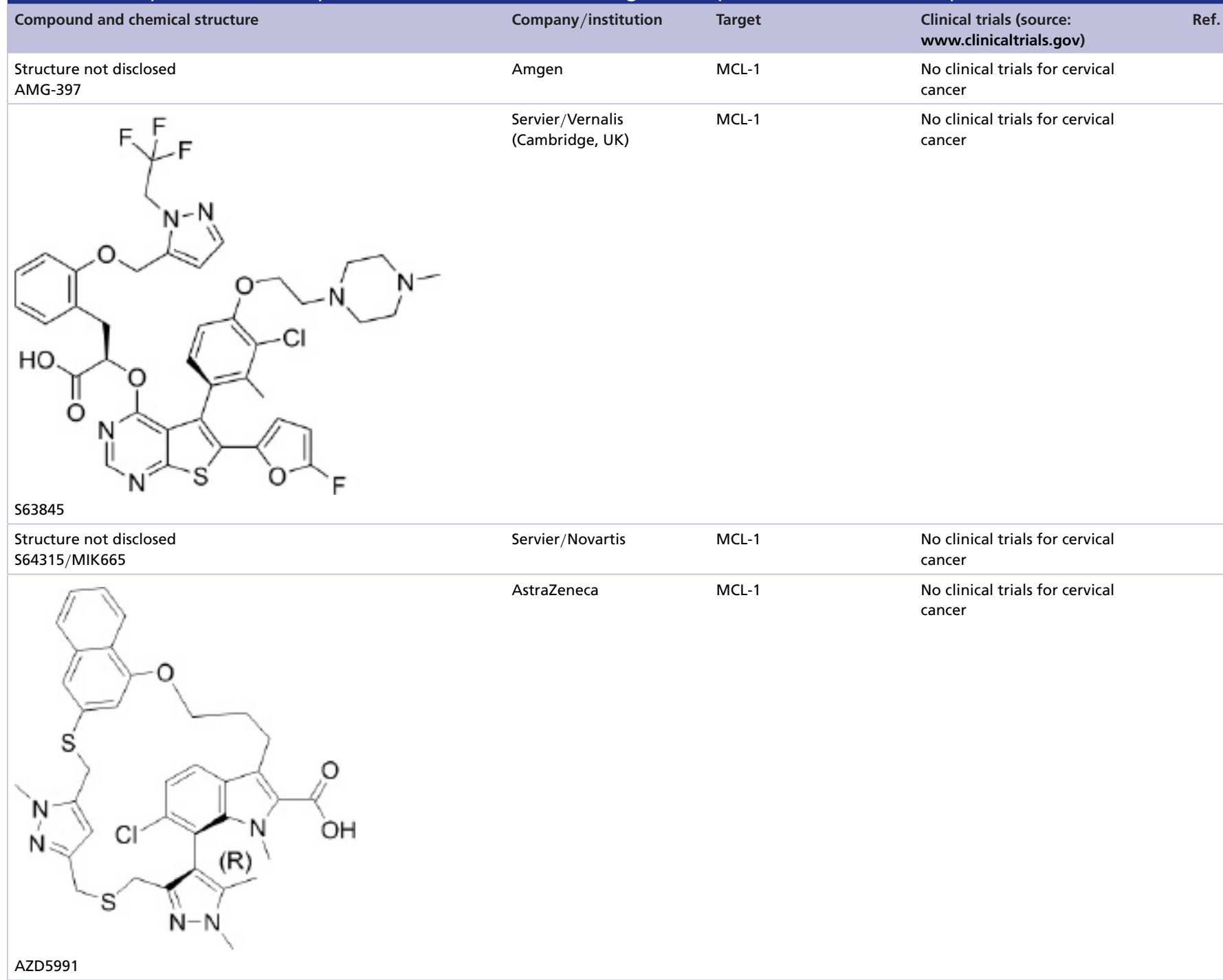

BCL-2: B-cell lymphoma 2.

A-1331852 were used to investigate the contributions of BCL-2 and BCL-XL in mediating cervical cancer cell survival. In order to study the role of MCL-1 for cell survival, MCL-1 selective inhibitor S63845, was employed. S63845 was reported to demonstrate higher affinity toward MCL-1 $(\mathrm{Ki}<1.2 \mathrm{nM})$ compared with A-1210477 $(\mathrm{Ki}=28 \mathrm{nM})$. In addition, S63845 was 1000-fold more potent in killing MCL-1 dependent H929 cells compared with A-1210477 [49]. In pilot experiments, when HeLa cells were treated singly with A-1210477, the cells were resistant to the treatment, with a $\mathrm{IC}_{50}$ value of $11.4 \pm 0.7 \mu \mathrm{M}$ (data not shown). However, when the same cells were tested with $S 63845$ singly, the cells were 11-fold more responsive to the drug $\left(\mathrm{IC}_{50}\right.$ : $\left.1.01 \pm 0.16 \mu \mathrm{M}\right)$, indicating that the cells depend on MCL-1 for survival. Given these findings, we reasoned that using a drug with higher affinity for MCL-1 would be more appropriate in helping delineate its role in cervical cancer cell survival.

In our subsequent study, four cervical cancer cell lines namely CaSki, HeLa, SiHa and C33A were treated with single agent ABT-199, A-1331852 and S63845. All four cell lines were resistant to single agent treatment of ABT-199 and A-1331852 indicating that the cells are neither BCL-2 nor BCL-XL-dependent for survival. Except for HeLa, all the other cell lines were resistant to single agent treatment of S63845, indicating that the cell lines also do not rely on MCL-1 for survival. Although HeLa cells were sensitive to single agent treatment of S63845, combination of ABT-199 or A-1331852 with concentrations of S63845 of $<1 \mu \mathrm{M}$ resulted in synergy, indicating that inhibition of either BCL-XL or BCL-2 is still required to achieve cell killing at lower drug concentrations of 


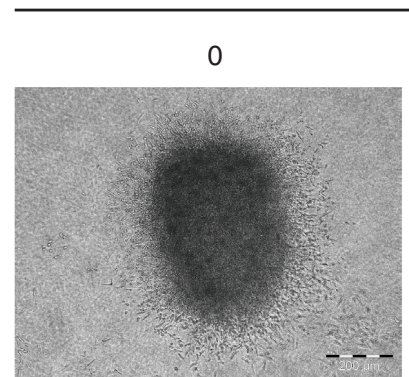

1
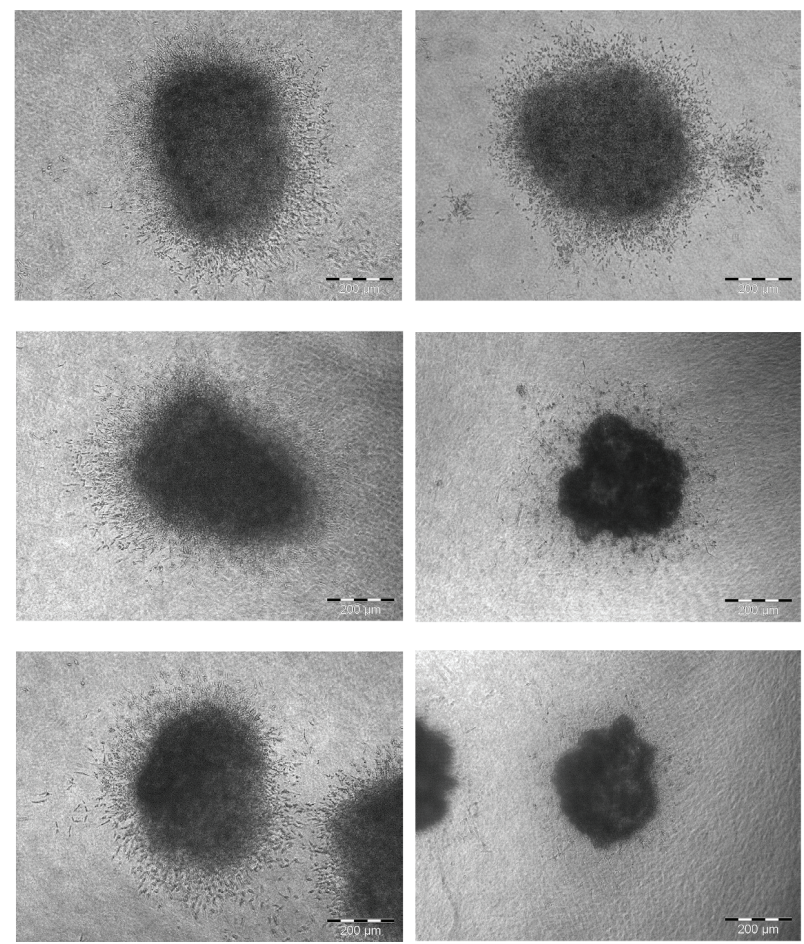

2
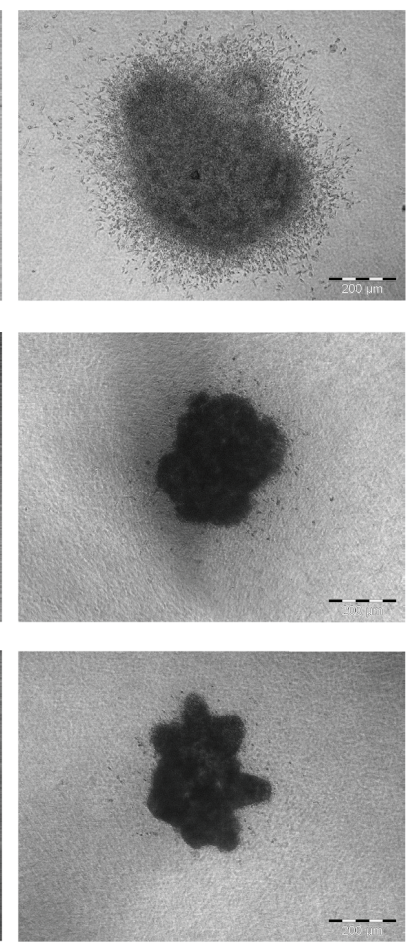

Figure 4. The effect of combination of MCL-1 selective inhibitor S63845 and BCL-XL selective inhibitor A-1331852 on the invasion of 3D spheroids generated from the cervical cancer cell line SiHa versus single agent treatment of S63845 and A-1331852 alone over $72 \mathrm{~h}$. The spheroids were treated with 563845 and A-1331852 over $72 \mathrm{~h}$ at indicated concentrations. Size bar: $200 \mu \mathrm{m}$.

S63845 [75]. Collectively, our data demonstrate that survival of the cervical cancer cell lines are maintained by more than one anti-apoptotic protein and selectively inhibiting them in combination kills the cells more effectively than drugs that inhibit one of these proteins alone.

Co-inhibition of BCL-XL and MCL-1 with A-1331852 and S63845 significantly inhibited cell proliferation of all four cell lines in 2D monolayer cultures and 3D spheroids [75]. Figure 4 demonstrates the effect of combination of A-1331852 and S63845 on the invasion of 3D spheroids generated from cervical cancer cell line SiHa. The 3D $\mathrm{SiHa}$ spheroids were treated with single agent A-1331852 and S63845 and combination of both drugs for $72 \mathrm{~h}$. The single agent treatment did not have an effect on the invasion of the spheroids. However, the combination showed an effect on the invasion of the spheroids. S63845 at $2 \mu \mathrm{M}$ was able to sensitize the spheroids to A-1331852, reflected in concentration-dependent inhibition of spheroid invasion. Similarly, A-1331852 at $2 \mu \mathrm{M}$ was able to sensitize the spheroids to S63845 (Figure 4).

Co-inhibition of BCL-2 and MCL-1 with ABT-199 and S63845, also inhibited cell proliferation of all cancer cell lines, except SiHa. However, the effect of combination of BCL-2 and MCL-1 was not as pronounced as combination of A-1331852 and S63845 [75]. Our findings were in accordance with other studies which reported that inhibition of BCL-XL rather than BCL-2 has resulted in sensitization of solid tumor cancer cell lines to other drugs. For example, osteosarcoma cells were sensitized to doxorubicin by BCL-XL inhibitor WEHI-539 but not BCL-2 inhibitors [76]. In another study, ABT-263 or BCL-XL selective inhibitors sensitized panels of breast cancer, non-small-cell lung cancer and ovarian cancer cell lines to docetaxel but not to BCL-2 inhibitors [73]. Similarly, chondrosarcoma cell lines were sensitized to doxorubicin or cisplatin by BCL-XL inhibitors and not by BCL-2 inhibitors [77]. Furthermore, lung squamous cell carcinoma cells were more susceptible to co-inhibition of BCL-XL 
and MCL-1 compared with inhibition of BCL-2 [78]. In a more recent study, drug combinations targeting BCL-XL and MCL-1, and to a lesser extent BCL-2 were reported to synergistically kill melanoma cells [79]. Collectively, solid tumors are more susceptible to inhibition of BCL-XL and MCL-1.

Given our findings, co-inhibition of these two proteins with appropriate combinations of $\mathrm{BH} 3$-mimetics would lower the threshold for apoptosis in cervical cancer cells resulting in apoptotic cell death.

\section{Future perspective}

Cervical cancer is treatable if diagnosed early; $80-90 \%$ of patients at early stage I and II and 60\% patients at stage II are treatable with the current treatment modalities namely surgery, chemo and radiotherapy [80]. The standard of care for patients with stage IVB or recurrent disease are cisplatin and paclitaxel. However, patients develop resistance to these first-line treatments and there are unfortunately limited second-line treatments available for these patients. So far, bevacuzimab (Avastin, Mvasi) and immune checkpoint inhibitor pembrolizumab (Keytruda) are the only targeted therapies approved by FDA for the treatment of advance and recurrent cervical cancer [7,81].

The BCL-2 anti-apoptotic proteins have gained a lot of attention as attractive therapeutic targets for cancer treatment. However, targeting these proteins for cervical cancer treatment were not extensively explored in spite of several studies reporting high expressions of these proteins in cervical cancer tissues. Our findings show that BCL-XL and MCL-1 are the key BCL-2 family proteins in cervical cancer cell survival. However, co-inhibiting BCL-XL and MCL-1 may cause an issue in the clinic, as inhibition of BCL-XL may result in thrombocytopenia, as platelets rely on BCL-XL for survival $[82,83]$. One strategy to overcome this issue, would be to develop dosing schedules which would employ lower doses of A-1331852 that would result in maximal cancer cell killing but not enough to cause thrombocytopenia. Given the findings of our study [74,75], thrombocytopenia may be able to be avoided, as all cervical cancer cell lines tested responded to low concentrations of combinations of A-1331852 and S63845. However, at present neither A-1331852 nor S63845 are tested in clinical trials, due to toxicity issues and co-targeting of BCL-XL and MCL-1 can cause fatal hepatotoxicity [78]. However, careful planning of dosing schedules, duration and route of drug administration may result in more favorable response in patients. Another strategy would be to either combine, less toxic BCL-XL or MCL-1 selective inhibitor with more conventional cytotoxic chemotherapy or treat cells with selective pro-apoptotic agents that directly activate type 2 mitochondrial pathways [84]. Co-targeting BCL-2 and MCL-1 would be another treatment avenue worth exploring for cervical cancer management. Although the effect of inhibiting BCL-2 was not as pronounced as inhibiting BCL-XL, we still observed synergy with combination of ABT-199 and S63845 in several cervical cancer cell lines [75]. Hence, combination of ABT-199 and S63845 could be an attractive therapeutic strategy worth exploring for cervical cancer management. The data presented in our studies suggest that combination of selective inhibitors of BCL-XL and MCL-1 or BCL-2 and MCL-1, are avenues worth exploring for cervical cancer. Moving forward, further testing, especially in animal models are needed to establish safety and efficacy profiles of these drugs. Nevertheless, the $\mathrm{BH} 3$-mimetics have the potential to be translated in clinic for cervical cancer management.

\section{Executive summary}

\section{The B-cell lymphoma 2 family proteins}

- The BCL-2 anti-apoptotic proteins are attractive targets for treatment and the efforts to target them increased with the development of BH3-mimetics.

Contribution of the BCL-2 anti-apoptotic proteins for cervical cancer progression

- Despite several studies reporting high expression levels of BCL-2 anti-apoptotic proteins in cervical cancer tissues, there are no considerable effort to target these proteins for cervical cancer management.

Targeting cervical cancer with BH3-mimetics

- Anti-apoptotic proteins MCL-1 and BCL-XL are crucial for the survival of cervical cancer cells and targeting these proteins with combination of BH3-mimetics, which selectively target MCL-1 and BCL-XL result in apoptotic cell death.

Future perspective

- Further investigation in animal models of cervical cancer are needed to establish the efficacy and toxicity profiles of the BH3-mimetics. Nevertheless, these small molecule inhibitors have the potential to be translated to clinic for cervical cancer treatment. 
Financial \& competing interests disclosure

This work was supported by the Ministry of Education Malaysia under Fundamental Research Grant Scheme (203/PBIOLOGI/6711541), L'Oréal Malaysia under L'Oréal -UNESCO FWIS (304/PBIOLOGI/650853/L117) and Universiti Sains Malaysia under Research University grant (1001/PBIOLOGI/8012268). The authors have no other relevant affiliations or financial involvement with any organization or entity with a financial interest in or financial conflict with the subject matter or materials discussed in the manuscript apart from those disclosed.

No writing assistance was utilized in the production of this manuscript.

\section{References}

Papers of special note have been highlighted as: $\bullet \bullet$ of considerable interest

1. Massad LS. Preinvasive disease of the cervix. In: Clinical Gyneocologic Oncology (9th Edition). DiSaia Philip, Creasman William, Mannel Robert, McMeekin D Scott, Mutch Davi. (Eds). Elsevier, PA, USA, 1-19 (2018).

2. Walboomers JM, Jacobs MV, Manos MM et al. Human papillomavirus is a necessary cause of invasive cervical cancer worldwide. J. Pathol. 189(1), 12-19 (1999).

3. Li Y, Xu C. Human papillomavirus-related cancers. In: Infectious Agents Associated Cancers: Epidemiology and Molecular Biology. Advances in Experimental Medicine and Biology. Cai Q, Yuan Z, Lan. (Eds). Springer, Singapore, 1018 (2017).

4. Bray F, Ferlay J, Soerjomataram I, Siegel RL, Torre LA, Jemal A. Global cancer statistics 2018: GLOBOCAN estimates of incidence and mortality worldwide for 36 cancers in 185 countries. CA Cancer J. Clin. 68(6), 394-424 (2018).

5. De Martel C, Plummer M, Vignat J, Franceschi S. Worldwide burden of cancer attributable to HPV by site, country and HPV type. Int. J. Cancer 141(4), 664-670 (2017).

6. Cohen PA, Jhingran A, Oaknin A, Denny L. Cervical cancer. Lancet 393(10167), 169-182 (2019).

-• Addresses the most recent treatment avenues available for cervical cancer management.

7. Johnson CA, James D, Marzan A, Armaos M. Cervical cancer: an overview of pathophysiology and management. Semin. Oncol. Nurs. 35(2), 166-174 (2019).

8. Ashkenazi A, Fairbrother WJ, Leverson JD, Souers AJ. From basic apoptosis discoveries to advanced selective BCL-2 family inhibitors. Nat. Rev. Drug Discov. 16(4), 273-284 (2017).

9. Mohana-Kumaran N, Hill DS, Allen JD, Haass NK. Targeting the intrinsic apoptosis pathway as a strategy for melanoma therapy. Pigment Cell Melanoma Res. 27(4), 525-539 (2014).

10. Ichim G, Tait SW. A fate worse than death: apoptosis as an oncogenic process. Nat. Rev. Cancer 16(8), 539-548 (2016).

11. Li H, Zhu H, Xu CJ, Yuan J. Cleavage of BID by caspase 8 mediates the mitochondrial damage in the Fas pathway of apoptosis. Cell 94(4), 491-501 (1998).

12. Montero J, Letai A. Why do BCL-2 inhibitors work and where should we use them in the clinic? Cell Death Differ. 25(1), 56-64 (2018).

-• Addresses the use of Dynamic BH3 Profiling dynamic BH3 profiling (DBP) for identifying cancer anti-apoptotic addiction.

13. Carpio MA, Michaud M, Zhou W, Fisher JK, Walensky LD, Katz SG. BCL-2 family member BOK promotes apoptosis in response to endoplasmic reticulum stress. Proc. Natl Acad. Sci. USA 112(23), 7201-7206 (2015).

14. Gross A, Jockel J, Wei MC, Korsmeyer SJ. Enforced dimerization of BAX results in its translocation, mitochondrial dysfunction and apoptosis. EMBO J. 17(14), 3878-3885 (1998).

15. Wei MC, Lindsten T, Mootha VK et al. tBID, a membrane-targeted death ligand, oligomerizes BAK to release cytochrome c. Genes Dev. 14(16), 2060-2071 (2000).

16. Inohara N, Ding L, Chen S, Nunez G. harakiri, a novel regulator of cell death, encodes a protein that activates apoptosis and interacts selectively with survival-promoting proteins Bcl-2 and Bcl-X(L). EMBO J. 16(7), 1686-1694 (1997).

17. Nakano K, Vousden KH. PUMA, a novel proapoptotic gene, is induced by p53. Mol. Cell 7(3), 683-694 (2001).

18. O'Connor L, Strasser A, O’Reilly LA et al. Bim: a novel member of the Bcl-2 family that promotes apoptosis. EMBO J. 17(2), 384-395 (1998).

19. Oda E, Ohki R, Murasawa $\mathrm{H}$ et al. Noxa, a BH3-only member of the $\mathrm{Bcl}-2$ family and candidate mediator of $\mathrm{p} 53$-induced apoptosis Science 288(5468), 1053-1058 (2000).

20. Wang K, Yin XM, Chao DT, Milliman CL, Korsmeyer SJ. BID: a novel BH3 domain-only death agonist. Genes Dev. 10(22), 2859-2869 (1996).

21. Yang E, Zha J, Jockel J, Boise LH, Thompson CB, Korsmeyer SJ. Bad, a heterodimeric partner for Bcl-XL and Bcl-2, displaces Bax and promotes cell death. Cell 80(2), 285-291 (1995).

22. Yu J, Zhang L, Hwang PM, Kinzler KW, Vogelstein B. PUMA induces the rapid apoptosis of colorectal cancer cells. Mol. Cell 7(3), 673-682 (2001). 
23. Delbridge AR, Grabow S, Strasser A, Vaux DL. Thirty years of BCL-2: translating cell death discoveries into novel cancer therapies. Nat. Rev. Cancer 16(2), 99-109 (2016).

24. Wolter KG, Hsu YT, Smith CL, Nechushtan A, Xi XG, Youle RJ. Movement of Bax from the cytosol to mitochondria during apoptosis. J. Cell Biol. 139(5), 1281-1292 (1997).

25. Merino D, Giam M, Hughes PD et al. The role of BH3-only protein Bim extends beyond inhibiting Bcl-2-like prosurvival proteins. J. Cell Biol. 186(3), 355-362 (2009).

26. Chen HC, Kanai M, Inoue-Yamauchi A et al. An interconnected hierarchical model of cell death regulation by the BCL-2 family. Nat. Cell Biol. 17(10), 1270-1281 (2015).

27. Dai H, Smith A, Meng XW, Schneider PA, Pang YP, Kaufmann SH. Transient binding of an activator BH3 domain to the Bak BH3-binding groove initiates Bak oligomerization. J. Cell Biol. 194(1), 39-48 (2011).

28. Kim H, Rafiuddin-Shah M, Tu HC et al. Hierarchical regulation of mitochondrion-dependent apoptosis by BCL-2 subfamilies. Nat. Cell Biol. 8(12), 1348-1358 (2006).

29. Antonsson B, Montessuit S, Sanchez B, Martinou JC. Bax is present as a high molecular weight oligomer/complex in the mitochondrial membrane of apoptotic cells. J. Biol. Chem. 276(15), 11615-11623 (2001).

30. Czabotar PE, Westphal D, Dewson G et al. Bax crystal structures reveal how BH3 domains activate Bax and nucleate its oligomerization to induce apoptosis. Cell 152(3), 519-531 (2013).

31. Mikhailov V, Mikhailova M, Degenhardt K, Venkatachalam MA, White E, Saikumar P. Association of Bax and Bak homo-oligomers in mitochondria. Bax requirement for Bak reorganization and cytochrome c release. J. Biol. Chem. 278(7), 5367-5376 (2003).

32. Certo M, Del Gaizo Moore V, Nishino M et al. Mitochondria primed by death signals determine cellular addiction to antiapoptotic BCL-2 family members. Cancer Cell 9(5), 351-365 (2006).

33. Cheng EH, Wei MC, Weiler $\mathrm{S}$ et al. BCL-2, BCL-X(L) sequester BH3 domain-only molecules preventing BAX-and BAK-mediated mitochondrial apoptosis. Mol. Cell 8(3), 705-711 (2001).

34. Letai A, Bassik MC, Walensky LD, Sorcinelli MD, Weiler S, Korsmeyer SJ. Distinct BH3 domains either sensitize or activate mitochondrial apoptosis, serving as prototype cancer therapeutics. Cancer Cell 2(3), 183-192 (2002).

35. Ding J, Mooers BH, Zhang Z et al. After embedding in membranes antiapoptotic Bcl-XL protein binds both Bcl-2 homology region 3 and helix 1 of proapoptotic Bax protein to inhibit apoptotic mitochondrial permeabilization. J. Biol. Chem. 289(17), 11873-11896 (2014).

36. Willis SN, Chen L, Dewson G et al. Proapoptotic Bak is sequestered by Mcl-1 and Bcl-xL, but not Bcl-2, until displaced by BH3-only proteins. Genes Dev. 19(11), 1294-1305 (2005).

37. Willis SN, Fletcher JI, Kaufmann T et al. Apoptosis initiated when BH3 ligands engage multiple Bcl-2 homologs, not Bax or Bak. Science 315(5813), 856-859 (2007).

38. Kuwana T, Bouchier-Hayes L, Chipuk JE et al. BH3 domains of BH3-only proteins differentially regulate Bax-mediated mitochondrial membrane permeabilization both directly and indirectly. Mol. Cell 17(4), 525-535 (2005).

39. Willis SN, Adams JM. Life in the balance: how BH3-only proteins induce apoptosis. Curr. Opin. Cell Biol. 17(6), 617-625 (2005).

40. Campbell KJ, Tait SWG. Targeting BCL-2 regulated apoptosis in cancer. Open Biol. 8(5), 180002 (2018).

41. Oltersdorf T, Elmore SW, Shoemaker AR et al. An inhibitor of Bcl-2 family proteins induces regression of solid tumours. Nature 435(7042), 677-681 (2005).

42. Tse C, Shoemaker AR, Adickes J et al. ABT-263: a potent and orally bioavailable Bcl-2 family inhibitor. Cancer Res. 68(9), 3421-3428 (2008).

43. Roberts AW, Seymour JF, Brown JR et al. Substantial susceptibility of chronic lymphocytic leukemia to BCL2 inhibition: results of a phase I study of navitoclax in patients with relapsed or refractory disease. J. Clin. Oncol. 30(5), 488-496 (2012).

44. Wilson WH, O'Connor OA, Czuczman MS et al. Navitoclax, a targeted high-affinity inhibitor of BCL-2, in lymphoid malignancies: a phase 1 dose-escalation study of safety, pharmacokinetics, pharmacodynamics, and antitumour activity. Lancet Oncol. 11(12), 1149-1159 (2010).

45. Souers AJ, Leverson JD, Boghaert ER et al. ABT-199, a potent and selective BCL-2 inhibitor, achieves antitumor activity while sparing platelets. Nat. Med. 19(2), 202-208 (2013).

46. Lessene G, Czabotar PE, Sleebs BE et al. Structure-guided design of a selective BCL-X(L) inhibitor. Nat. Chem. Biol. 9(6), 390-397 (2013).

47. Leverson JD, Zhang H, Chen J et al. Potent and selective small-molecule MCL-1 inhibitors demonstrate on-target cancer cell killing activity as single agents and in combination with ABT-263 (navitoclax). Cell Death Dis. 6, e1590 (2015).

48. Tao ZF, Hasvold L, Wang L et al. Discovery of a potent and selective BCL-XL Inhibitor with in vivo activity. ACS Med. Chem. Lett. 5(10), 1088-1093 (2014).

49. Kotschy A, Szlavik Z, Murray J et al. The MCL1 inhibitor S63845 is tolerable and effective in diverse cancer models. Nature 538(7626), 477-482 (2016). 
50. Hird AW, Tron AE. Recent advances in the development of Mcl-1 inhibitors for cancer therapy. Pharmacol. Ther. 198, 59-67 (2019).

- Addresses the most recent MCL-1 agonists for research and therapy.

51. Caenepeel S, Brown SP, Belmontes B et al. AMG 176, a selective MCL1 inhibitor, is effective in hematologic cancer models alone and in combination with established therapies. Cancer Discov. 8(12), 1582-1597 (2018).

52. Johannes JW, Bates S, Beigie C et al. Structure based design of non-natural peptidic macrocyclic Mcl-1 inhibitors. ACS Med. Chem. Lett. 8(2), 239-244 (2017).

53. Tron AE, Belmonte MA, Adam A et al. Discovery of Mcl-1-specific inhibitor AZD5991 and preclinical activity in multiple myeloma and acute myeloid leukemia. Nat. Commun. 9(1), 5341 (2018).

54. Hanahan D, Weinberg RA. Hallmarks of cancer: the next generation. Cell 144(5), 646-674 (2011).

55. Touzeau C, Maciag P, Amiot M, Moreau P. Targeting Bcl-2 for the treatment of multiple myeloma. Leukemia 32(9), 1899-1907 (2018).

56. Touzeau C, Ryan J, Guerriero J et al. BH3 profiling identifies heterogeneous dependency on Bcl-2 family members in multiple myeloma and predicts sensitivity to BH3 mimetics. Leukemia 30(3), 761-764 (2016).

57. Lin KH, Winter PS, Xie A et al. Targeting MCL-1/BCL-XL forestalls the acquisition of resistance to ABT-199 in acute myeloid leukemia. Sci. Rep. 6, 27696 (2016).

58. Peperzak V, Slinger E, Ter Burg J, Eldering E. Functional disparities among BCL-2 members in tonsillar and leukemic B-cell subsets assessed by BH3-mimetic profiling. Cell Death Differ. 24(1), 111-119 (2017).

59. Li Z, He S, Look AT. The MCL1-specific inhibitor $\$ 63845$ acts synergistically with venetoclax/ABT-199 to induce apoptosis in T-cell acute lymphoblastic leukemia cells. Leukemia 33(1), 262-266 (2019).

60. Grace VM, Shalini JV, Lekha TT, Devaraj SN, Devaraj H. Co-overexpression of $\mathrm{p} 53$ and bcl-2 proteins in HPV-induced squamous cell carcinoma of the uterine cervix. Gynecol. Oncol. 91(1), 51-58 (2003).

61. Dimitrakakis C, Kymionis G, Diakomanolis E et al. The possible role of $\mathrm{p} 53$ and bcl-2 expression in cervical carcinomas and their premalignant lesions. Gynecol. Oncol. 77(1), 129-136 (2000).

62. Shukla S, Dass J, Pujani M. p53 and bcl2 expression in malignant and premalignant lesions of uterine cervix and their correlation with human papilloma virus 16 and 18. South Asian J. Cancer 3(1), 48-53 (2014).

63. Tjalma W, De Cuyper E, Weyler J et al. Expression of bcl-2 in invasive and in situ carcinoma of the uterine cervix. Am. J. Obstet. Gynecol. 178(1 Pt 1), 113-117 (1998).

64. Wootipoom V, Lekhyananda N, Phungrassami T, Boonyaphiphat P, Thongsuksai P. Prognostic significance of Bax, Bcl-2, and p53 expressions in cervical squamous cell carcinoma treated by radiotherapy. Gynecol. Oncol. 94(3), 636-642 (2004).

65. Zhou XL, Wang M. Expression levels of survivin, Bcl-2, and KAI1 proteins in cervical cancer and their correlation with metastasis. Genet. Mol. Res. 14(4), 17059-17067 (2015).

66. Aletra C, Ravazoula P, Scopa C et al. Expression of bcl-2 and bax in cervical intraepithelial neoplasia and invasive squamous cell carcinoma of the uterine cervix. Eur. J. Gynaecol. Oncol. 21(5), 494-498 (2000).

67. Zhang T, Zhao C, Luo L, Zhao H, Cheng J, Xu F. The expression of Mcl-1 in human cervical cancer and its clinical significance. Med. Oncol. 29(3), 1985-1991 (2012).

68. Tuohetimulati G, Zhu M, Chen J, Niyazi M. Expressions and clinical significance of Bcl-2, Bcl-xL and c-IAP1 protein in cervical cancer. Int. J. Clin. Exp. Med. 11(11), 12361-12367 (2018).

69. Park IC, Lee SH, Whang DY et al. Expression of a novel Bcl-2 related gene, Bfl-1, in various human cancers and cancer cell lines. Anticancer Res. 17(6D), 4619-4622 (1997).

70. Merino D, Kelly GL, Lessene G, Wei AH, Roberts AW, Strasser A. BH3-mimetic drugs: blazing the trail for new cancer medicines. Cancer Cell 34(6), 879-891 (2018).

-. Addresses the most recent advances in the development of BH3-mimetics for therapy.

71. Ramsey HE, Fischer MA, Lee T et al. A novel MCL1 inhibitor combined with venetoclax rescues venetoclax-resistant acute myelogenous leukemia. Cancer Discov. 8(12), 1566-1581 (2018).

72. Aubrey BJ, Kelly GL, Kueh AJ et al. An inducible lentiviral guide RNA platform enables the identification of tumor-essential genes and tumor-promoting mutations in vivo. Cell Rep. 10(8), 1422-1432 (2015).

-• Addresses the use of CRISPR/Cas9 technique for identifying cancer anti-apoptotic addiction.

73. Leverson JD, Phillips DC, Mitten MJ et al. Exploiting selective BCL-2 family inhibitors to dissect cell survival dependencies and define improved strategies for cancer therapy. Sci. Transl. Med. 7(279), 279ra240 (2015).

-. Addresses the use of BH3-mimetics as chemical toolkit for identifying cancer anti-apoptotic addiction.

74. Lian BSX, Yek AEH, Shuvas H, Abdul Rahman SF, Muniandy K, Mohana-Kumaran N. Synergistic anti-proliferative effects of combination of ABT-263 and MCL-1 selective inhibitor A-1210477 on cervical cancer cell lines. BMC Res. Notes 11(1), 197 (2018).

-• Addresses preclinical investigations of combination of BH3-mimetics ABT-263 and A-1210477 in cervical cancer cell lines. 
75. Abdul Rahman SF, Muniandy K, Yong Kit Soo et al. Co-inhibition of BCL-XL and MCL-1 with selective BCL-2 family inhibitors enhances cytotoxicity of cervical cancer cell lines. Biochem. Biophys. Rep. 22, 100756 (2020).

-. Highlights the importance of targeting anti-apoptotic proteins MCL-1 and BCL-XL in cervical cancer cell lines.

76. Baranski Z, De Jong Y, Ilkova T et al. Pharmacological inhibition of Bcl-xL sensitizes osteosarcoma to doxorubicin. Oncotarget 6(34), 36113-36125 (2015).

77. De Jong Y, Van Maldegem AM, Marino-Enriquez A et al. Inhibition of Bcl-2 family members sensitizes mesenchymal chondrosarcoma to conventional chemotherapy: report on a novel mesenchymal chondrosarcoma cell line. Lab. Invest. 96(10), 1128-1137 (2016).

78. Weeden CE, Ah-Cann C, Holik AZ et al. Dual inhibition of BCL-XL and MCL-1 is required to induce tumour regression in lung squamous cell carcinomas sensitive to FGFR inhibition. Oncogene 37(32), 4475-4488 (2018).

79. Lee EF, Harris TJ, Tran S et al. BCL-XL and MCL-1 are the key BCL-2 family proteins in melanoma cell survival. Cell Death Dis. 10(5), 342 (2019).

80. Fuentas A, Garcia AA. Advancements in cervical cancer prevention and management of persistent, recurrent, and metastatic disease: 2016 update. Am. J. Hematol. Oncol. 12(12), 8-17 (2016).

81. Vora C, Gupta S. Targeted therapy in cervical cancer. ESMO Open 3(Suppl. 1), e000462 (2018).

82. Zhang H, Nimmer PM, Tahir SK et al. Bcl-2 family proteins are essential for platelet survival. Cell Death Differ. 14(5), $943-951$ (2007).

83. Mason KD, Carpinelli MR, Fletcher JI et al. Programmed anuclear cell death delimits platelet life span. Cell 128(6), 1173-1186 (2007).

84. Quayle LA, Pereira MG, Scheper G et al. Anti-angiogenic drugs: direct anti-cancer agents with mitochondrial mechanisms of action. Oncotarget 8(51), 88670-88688 (2017). 
